\title{
Laser gain and threshold properties in compressive-strained and lattice-matched GaInNAs/GaAs quantum wells
}

\author{
W. W. Chow, E. D. Jones, N. A. Modine, A. A. Allerman and S. R. Kurtz \\ Sandia National Laboratories \\ Albuquerque, NM 85718-0601, U. S. A.
}

(July 13, 1999)

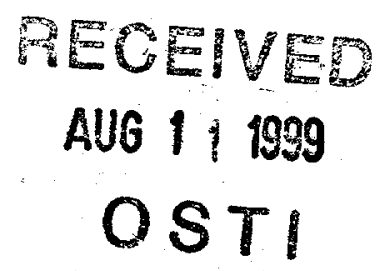

The optical gain spectra for compressive-strained and lattice-matched GaInNAs/GaAs quantum wells are computed using a microscopic laser theory. From these spectra, the peak gain and carrier radiative decay rate as functions of carrier density are determined. These dependences allow the study of lasing threshold current density for different GaInNAs/GaAs laser structures. 


\section{DISCLAIMER}

This report was prepared as an account of work sponsored by an agency of the United States Government. Neither the United States Government nor any agency thereof, nor any of their employees, make any warranty, express or implied, or assumes any legal liability or responsibility for the accuracy, completeness, or usefulness of any information, apparatus, product, or process disclosed, or represents that its use would not infringe privately owned rights. Reference herein to any specific commercial product, process, or service by trade name, trademark, manufacturer, or otherwise does not necessarily constitute or imply its endorsement, recommendation, or favoring by the United States Government or any agency thereof. The views and opinions of authors expressed herein do not necessarily state or reflect those of the United States Government or any agency thereof. 


\section{DISCLAIMER}

Portions of this document may be illegible in electronic image products. Images are produced from the best available original document. 
GaInNAs quantum wells were recently demonstrated as gain media for diode lasers. [1] Advantages of the GaInNAs alloy system include emission at wavelengths important for fiber optic communications, and close lattice match to GaAs. Since lasers based on this material system are in an early developmental stage, there are many questions concerning optical properties, expected device performance, and optimal laser configuration. This paper addresses some of these issues from a theoretical aspect. We begin with a discussion of material parameters needed in GaInNAs/GaAs quantum well bandstructure calculations. The electron and hole energy dispersions, and optical transition matrix elements from these calculations are used in computing the gain and spontaneous emission spectra. From the carrier density dependences of these spectra, we obtain the necessary gain vs current density relations.

We consider the quantum well structures, $\mathrm{Ga}_{0.93} \mathrm{In}_{0.07} \mathrm{~N}_{0.02} \mathrm{As}_{0.98} / \mathrm{GaAs}$ and $\mathrm{Ga}_{0.7}$ $\operatorname{In}_{0.3} \mathrm{~N}_{0.004} \mathrm{As}_{0.996} / \mathrm{GaAs}$. The latter is used in earlier GaInNAs lasers, [1] and is compressively strained. The former is being investigated for laser and solar cell applications. [2] A unique property of this structure is that $\mathrm{Ga}_{0.93} \mathrm{In}_{0.07} \mathrm{~N}_{0.02} \mathrm{As}_{0.98}$ has a bandgap energy around $\mathrm{leV}$, and yet is lattice matched to GaAs! While compressive strain in general improves laser properties, the lattice-matched structure allows the growth of thick active layers (as in epilayers and superlattices) that improve spatial overlap with the optical field, which is important in solar cell and some laser applications. The two structures are also chosen to illustrate strain effects on optical properties in the GaInNAs/GaAs system. While some of the strain effects will be similar to those in other semiconductor lasers, we expect important differences as well. One difference is the relatively large change in the electron effective mass due to the presence of nitrogen, which in turn changes the optical dipole matrix element and bandfilling effects. Here, we show that significant differences can result in the carrier density and current density dependences of gain, between the two aforementioned GaInNAs/GaAs structures, as well as between these structures and those involving GaInAs/InP, a commonly used material system for long-wavelength semiconductor lasers.

Our analysis begins with the compilation of needed GaInNAs material parameters. For 
small nitrogen concentrations, most of the $\mathrm{Ga}_{1-x} \operatorname{In}_{x} \mathrm{~N}_{y} \mathrm{As}_{1-y}$ material parameters may be approximated by the corresponding ones for $\mathrm{Ga}_{1-x} \mathrm{In}_{x}$ As. [3] Exceptions are the bandgap energy and electron effective mass. [4] To obtain expressions for the dependences of these two quantities on nitrogen concentration, we use the following approach. From room temperature photoluminescence experiments, the bandgap energy for $\mathrm{Ga}_{0.93} \mathrm{In}_{0.07} \mathrm{~N}_{0.02} \mathrm{As}_{0.98}$ is $\varepsilon_{g 0}=1.05 \mathrm{eV} .[5]$ With this information and a schematic diagram for the band lineup, [1] we propose the phenomenological relationship

$$
\varepsilon_{g 0}\left(\mathrm{Ga}_{1-x} \operatorname{In}_{x} \mathrm{~N}_{y} \mathrm{As}_{1-y}\right)=\varepsilon_{g 0}\left(\mathrm{Ga}_{1-x} \operatorname{In}_{x} \mathrm{As}\right)-69 e V \times \Delta e(x, y)
$$

where $\Delta e(x, y)=e(x, y)-e(x, 0)$ is the difference between the strain computed for $G a_{1-x} I n_{x} N_{y} A s_{1-y}$ and $G a_{1-x} I n_{x} A s, e(x, y)=[a(0,0)-a(x, y)] / a(x, y)$, and $a(x, y)$ is the lattice constant for $\mathrm{Ga}_{1-x} \operatorname{In}_{x} \mathrm{~N}_{y} \mathrm{As}_{1-y}$. We have also found for $\mathrm{Ga}_{0.93} \mathrm{In}_{0.07} \mathrm{~N}_{0.02} \mathrm{As}_{0.98}$ the electron mass $m_{e}=0.14 m_{0}$, where $m_{0}$ is the free electron mass. [5] This unusually heavy (compared to GaAs) electron mass is verified by different measurement techniques, [6] and by a first principles bandstructure calculation. [5] Using this data point in a schematic diagram similar to Eq. (1)

$$
m_{e}\left(\mathrm{Ga}_{1-x} \operatorname{In}_{x} \mathrm{~N}_{y} \mathrm{As}_{1-y}\right)=m_{e}\left(\mathrm{Ga}_{1-x} \operatorname{In}_{x} \mathrm{As}\right)+18.1667 m_{0} \Delta e(x, y)
$$

for an alloy of arbitrary composition. The values of $\varepsilon_{g 0}$ and $m_{e}$ calculated for $\mathrm{Ga}_{0.7} \operatorname{In}_{0.3} \mathrm{~N}_{0.004} \mathrm{As}_{0.996}$ and $\mathrm{Ga}_{0.7} \operatorname{In}_{0.3} \mathrm{~N}_{0.004} \mathrm{As}_{0.996}$, together with the other parameters needed for the quantum well bandstructure calculations are listed in Table I.

Based on results from bandstructure calculations involving the bulk alloy, [5] we assume in the $\mathrm{Ga}_{1-x} \operatorname{In}_{x} \mathrm{~N}_{y} \mathrm{As}_{1-y} / \mathrm{GaAs}$ quantum well bandstructure calculations that the presence of nitrogen affects mainly the conduction band. Then, to a good approximation, we can compute the hole bandstructure by performing a $\vec{k} \cdot \vec{p}$ calculation for a $\mathrm{Ga}_{1-x} \mathrm{In}_{x} \mathrm{As}-\mathrm{GaAs}$ quantum well structure, where we assume that the strain is $e(x, y)$, i.e., similar to that in $\mathrm{Ga}_{1-x} \operatorname{In}_{x} \mathrm{~N}_{y} \mathrm{As}_{1-y} / \mathrm{GaAs}$. For the lattice-matched structure, the hole bandstructure consists of two heavy hole subbands (hh1 and hh2) and one light hole subband (lh1). In contrast, 
only the heavy hole is Type 1 in the compressive-strained structure, leading to much higher hole band curvatures. There is also a significant difference in the electron effective masses, as may be seen from Table 1.

To investigate the bandstructure influence on optical properties, we calculate the gain spectra for different carrier densities. We use an approach based on the semiconductor Bloch equations, $[7,8]$ because it has several advantages that are important to our study. Unlike the more familiar gain calculations based on the relaxation rate approximation, our approach treats carrier-carrier collisions at the level of quantum kinetic equations. This leads to more precise predictions of gain properties, because the dephasing rate is eliminated as a free parameter. Equally important, our approach includes the Coulomb correlation contributions that are important for accurate descriptions of experimental gain and absorption spectra. [8]

Figure 1 shows the room temperature TE and TM gain spectra for the lattice-matched quantum well at different carrier densities. The two polarizations describe electric field polarization in (TE) and perpendicular to (TM) the plane of the quantum well. For the same carrier density, the spectra show approximately equal gain in the two polarizations, which is typical for unstrained quantum wells. The two absorption resonances in the TE spectrum for $N=5 \times 10^{11} \mathrm{~cm}^{-1}$ arise from the e1-hh1 and e1-lh1 transitions. Figure 2 shows noticeably different behaviors for the compressive-strained quantum well. Because the structure contains only heavy hole bound states, the TM absorption and gain are highly attenuated. Compared to the lattice-matched structure, the compressive-strained quantum well has significantly lower transparency carrier density and higher differential gain $d G / d N$. Figure $3 a$ clearly shows these differences due to differences in band curvatures. The higher electron and hole curvatures in the compressive-strained structure lead to smaller joint densities of states, which in turn make the creation of a population inversion easier. For comparison, we also plotted the curve for a $5 \mathrm{~nm} \mathrm{Ga}_{0.67} \operatorname{In}_{0.33} \mathrm{As}-\mathrm{InP}$ quantum well, which is representative of a more developed material system for long-wavelength semiconductor lasers. To obtain an emission energy that is approximately that of the other structures, we 
choose an In concentration of 0.33 , which results in an $e=0.02$ tensile strained quantum well. As the hole curvatures for the lattice-matched GaInNAs/GaAs and the tensile strained GaInAs/InP quantum wells are approximately similar, the difference in the transparency carrier densities for these two structures may be traced to the significantly larger electron effective mass in the former.

In order to study threshold current behavior, we need in addition to the curves in Fig. 3a, the radiative carrier recombination rate $w_{s p}$. To calculate this rate, we use a phenomenological relationship that relates the spontaneous emission and gain spectra. [9] Figure 3b shows $w_{s p}$ vs carrier density for the three gain structures. Figure 4 combines the results of Figs. $3 \mathrm{a}$ and $3 \mathrm{~b}$ to obtain the dependence of peak gain on the spontaneous emission current density, $J_{s p}=e w w_{s p}$, where $e$ is the electron charge, and $w$ is the quantum well width. The curves give the theoretical limit to the threshold current density for threshold gain $G_{t h}=G_{p k}$. An interesting result is that in spite of the large difference in transparency carrier density between the two GaInNAs/GaAs quantum wells, they have approximately the same transparency current densities, suggesting partial cancellation of bandstructure differences in a gain vs current density curve. Above transparency, bandstructure effects still play important roles, e.g., in giving a greater $\mathrm{dG}_{p k} / \mathrm{dJ}_{s p \text {. }}$ to the compressive-strained structure. For a typical threshold gain of $G_{t h}=10^{3} \mathrm{~cm}^{-1}$, this greater slope results in a threshold current density for the compressive-strained quantum well (TE polarization) that is half of that for lattice-matched one (either polarization).

Comparison of the GaInAs/InP and GaInNAs/GaN curves in Figs. 3a and 4 illustrates another mechanism that causes differences in behavior between $G_{p k}$ vs $N$ and $G_{p k}$ vs $J_{s p}$. In Fig. 3a, we note that the transparency carrier density is greater in the lattice-matched GaInNAs/GaN quantum well than in GaInAs-InP. Figure 4 shows the reverse, with GaInAs/InP having the higher transparency current density. The explanation for this behavior is the difference between the dipole matrix elements. Because the bulk material dipole matrix element is inversely proportional to the electron effective mass, the GaInAs/InP structure has a bulk material dipole matrix element that is twice that of GaInNAs/GaAs. The trans- 
parency carrier density depends primarily on bandfilling effects and thus is sensitive to band curvatures, but not to the dipole matrix element. On the other hand, the radiative carrier recombination rate $w_{s p}$ is proportional to the square of the dipole matrix element, and the larger dipole matrix element in GaInAs/InP leads to greater spontaneous emission loss, and consequently to a higher transparency current density. The spontaneous emission losses at transparency for the three quantum well structures are shown by the dots in Fig. 3b.

In summary, we use a many-body microscopic laser theory to investigate the gain and threshold current properties of GaInNAs/GaAs quantum wells. The calculations show gain properties that depend significantly on strain. Some of the strain effects are similar to those in other semiconductor lasers, especially effects arising from modifications to the hole bandstructure. However, the strain effects arising from conduction band and optical matrix element modifications are unique to the GaInNAs/GaAs system. These effects strongly influence the gain vs carrier density and gain vs current density relations.

This work was supported in part by the U. S. Department of Energy under contract No. DE-AC04-94AL85000.

Sandia is a multiprogram laboratory operated by Sandia Corporation, a Lockheed Martin Company. for the United States Department of Energy under contract DE-ACO4-94AL85000. 


\section{References}

[1] M. Kondow, T. Kitatani, S. Nakatsuka, M. Larson, K. Nakahara, Y. Yazawa, M. Okai and K. Uomi, IEEE J. Selected Topics in Quantum Electron. 3, 719 (1997).

[2] S. R. Kurtz, A. A. Allerman, E. D. Jones, J. M. Gee, J. J. Banas and B. E. Hammons, Appl. Phys. Lett. (to be published).

[3] S. Sakai and T. Abe, Extd. Abstr. 41st Spring Meeting Jpn., Soc. Appl. Phys., Tokyo, 186 (1994).

[4] W. G. Bi and C. W. Tu, Appl. Phys. Lett. 70, 1608 (1999).

[5] E. D. Jones, N. A. Modine, A. A. Allerman, I. J. Fritz, S. R. Kurtz, A. F. Wright, S. T. Tozer and X. Wei, Proc. SPIE 3621, 52 (1999); E. D. Jones, N. A. Modine, A. A. Allerman, S. R. Kurtz, A. F. Wright, S. T. Tozer and X. Wei, Phys. Rev B 60, 1 (1999).

[6] E. D. Jones, S. T. Tozer and T. Schmiedel, Physica E 176, 284 (1997).

[7] W. W. Chow, A.F. Wright, A. Girndt, F. Jahnke and S. W. Koch, Appl. Phys. Lett. 71, 2608 (1997)

[8] For a text book discussion see: W. W. Chow and S. W. Koch, Semiconductor-Laser Fundamentals: Physics of the Gain Materials (Springer, Berlin, 1999).

[9] C. H. Henry, R. A. Logan and F. R. Merritt, J. Appl. Phys. 51, 3042 (1980). 
Table I. Material parameters for lattice-matched $(e=0)$ Ga0.93 $\operatorname{In}_{0.07} \mathrm{~N}_{0.02} \mathrm{As}_{0.98}$ and compressive-strained $(e=-0.02) \mathrm{Ga}_{0.7} \operatorname{In}_{0.3} \mathrm{~N}_{0.004} \mathrm{As}_{0.996} . \Gamma_{1}, \Gamma_{2}$ and $\Gamma_{3}$ are the Luttinger parameters, $v_{b e}, v_{b h h}$, and $v_{b l h}$ are the electron, heavy-hole and light-hole band offsets, $\mu$ is the bulk material dipole moment matrix element, $\mathrm{C}_{11}$ and $\mathrm{C}_{12}$ are the elastic constants, $a$ is the lattice constant, $a_{1}$ and $a_{2}$ are the deformation potentials .

\begin{tabular}{|c|c|c|c|c|c|}
\hline & \multicolumn{2}{|c|}{$e=0 e=-.02$} & \multicolumn{3}{|c|}{$e=0 e=-.02$} \\
\hline $\mathrm{m}_{e} / \mathrm{m}_{0}$ & 0.14 & 0.069 & $\mathrm{v}_{b l h}(\mathrm{eV})$ & 0.028 & -0.023 \\
\hline$\Gamma_{1}$ & 7.75 & 10.71 & $\mu / e(\AA)$ & 3.3 & 5.5 \\
\hline$\Gamma_{2}$ & 2.54 & 3.99 & $\mathrm{a}(\AA)$ & 5.66 & 5.77 \\
\hline$\Gamma_{3}$ & 3.35 & 4.82 & $\mathrm{C}_{11}(\mathrm{GPa})$ & 116 & 108 \\
\hline$\varepsilon_{g 0}(\mathrm{eV})$ & 1.050 & 1.027 & $\mathrm{C}_{12}(\mathrm{GPa})$ & 53 & 51 \\
\hline $\mathrm{v}_{b e}(\mathrm{eV})$ & 0.339 & 0.283 & $a_{1}(e V)$ & -7.02 & -6.74 \\
\hline $\mathrm{v}_{b h h}(\mathrm{eV})$ & 0.033 & 0.113 & $a_{2}(e V)$ & -1.71 & -1.73 \\
\hline
\end{tabular}




\section{Figure Captions}

Figure 1. Room temperature (a) TE and (b) TM gain spectra for lattice-matched $7 \mathrm{~nm}$ $\mathrm{Ga}_{0.93} \mathrm{In}_{0.07} \mathrm{~N}_{0.02} \mathrm{As}_{0.98} / \mathrm{GaAs}$ and carrier densities $N=0.5,1,2,3,4,6$ and $8 \times 10^{12} \mathrm{~cm}^{-2}$.

Figure 2. Room temperature TE (solid curves) and TM (dashed curves) gain spectra for compressive-strained $7 n m \mathrm{Ga}_{0.7} \mathrm{In}_{0.3} \mathrm{~N}_{0.004} \mathrm{As}_{0.996} / \mathrm{GaAs}$ quantum well and carrier densities $N=0.1,0.5,1.0,1.5,2.0$, and $3 \times 10^{12} \mathrm{~cm}^{-2}$.

- Figure 3. (a) Peak gain versus carrier density for TE (solid curve) and TM (dashed curve) polarization in lattice-matched GaInNAs/GaAs, TE polarization in compressive-strained GaInNAs/GaAs (dot-dashed curve), and TM polarization in tensile-strained GaInAs/InP (dotted curve). (b) Radiative carrier recombination rate vs carrier density for lattice-matched (solid curve) and compressive-strained (dot-dashed curve) GaInNAs-GaAs, and tensile-strained GaInAs/InP (dotted curve).

Figure 4 Peak gain versus spontaneous emission current density for the three structures in Fig. 3. The notation is similar to the that in Figure 3a. 

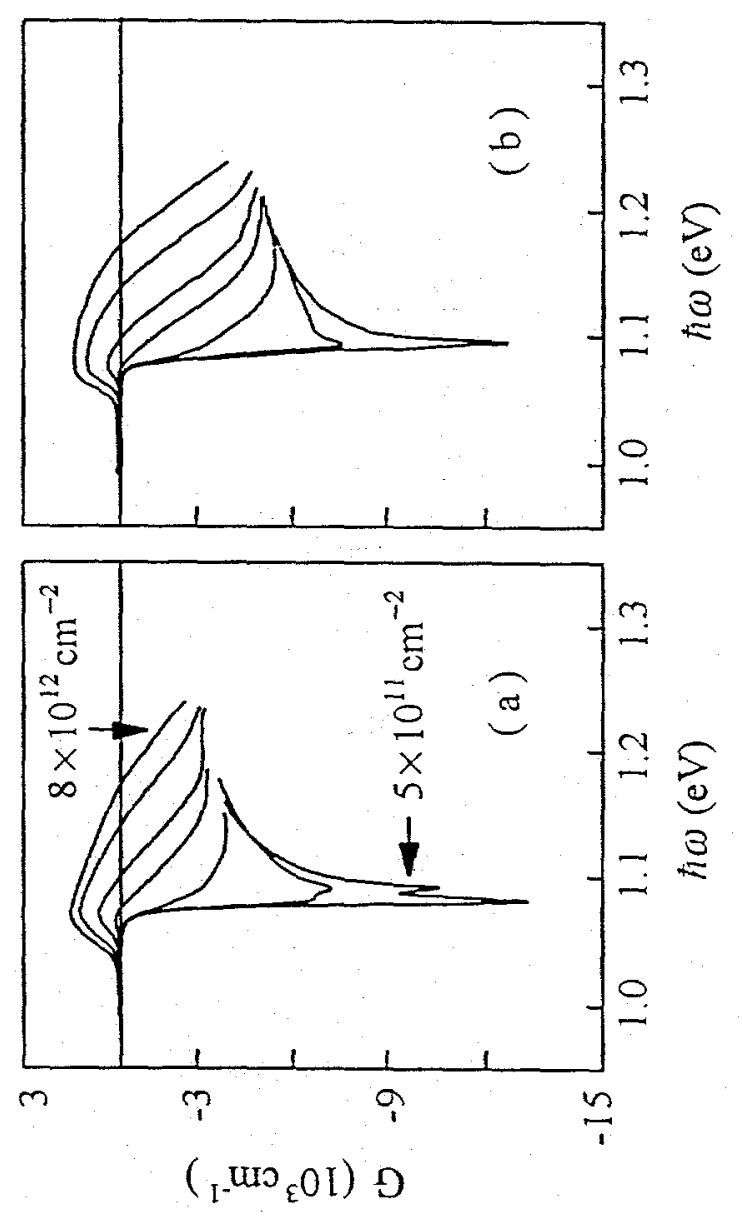


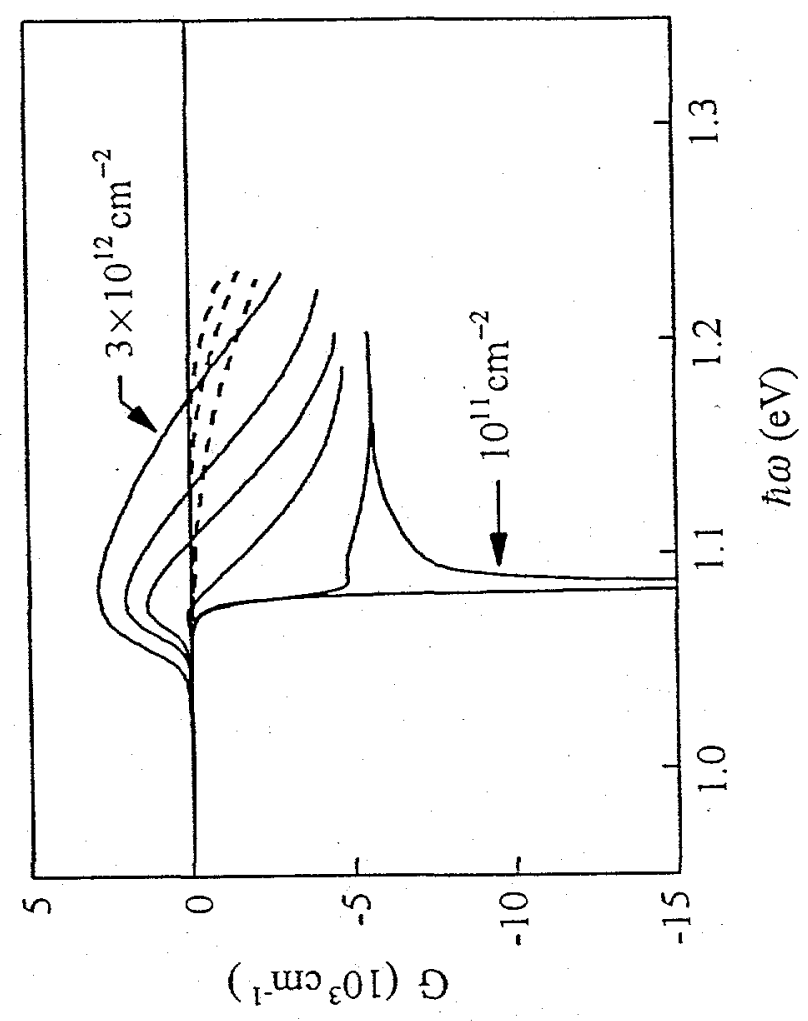

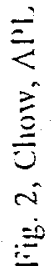



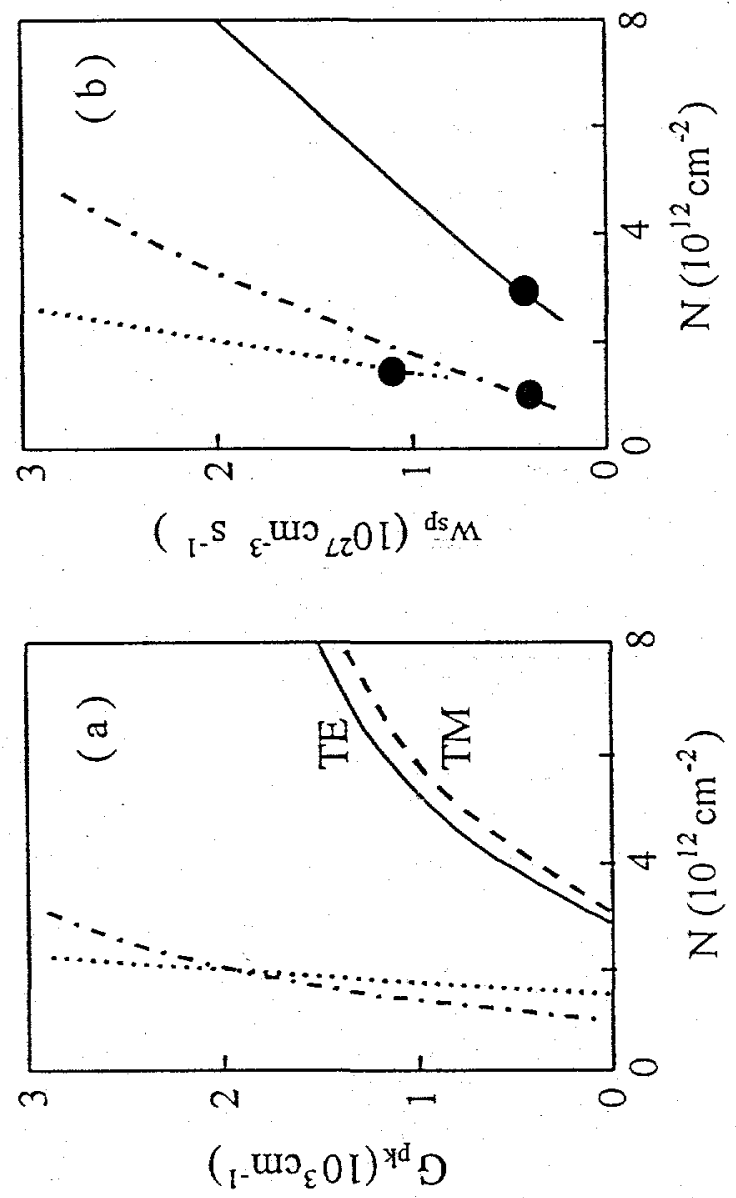


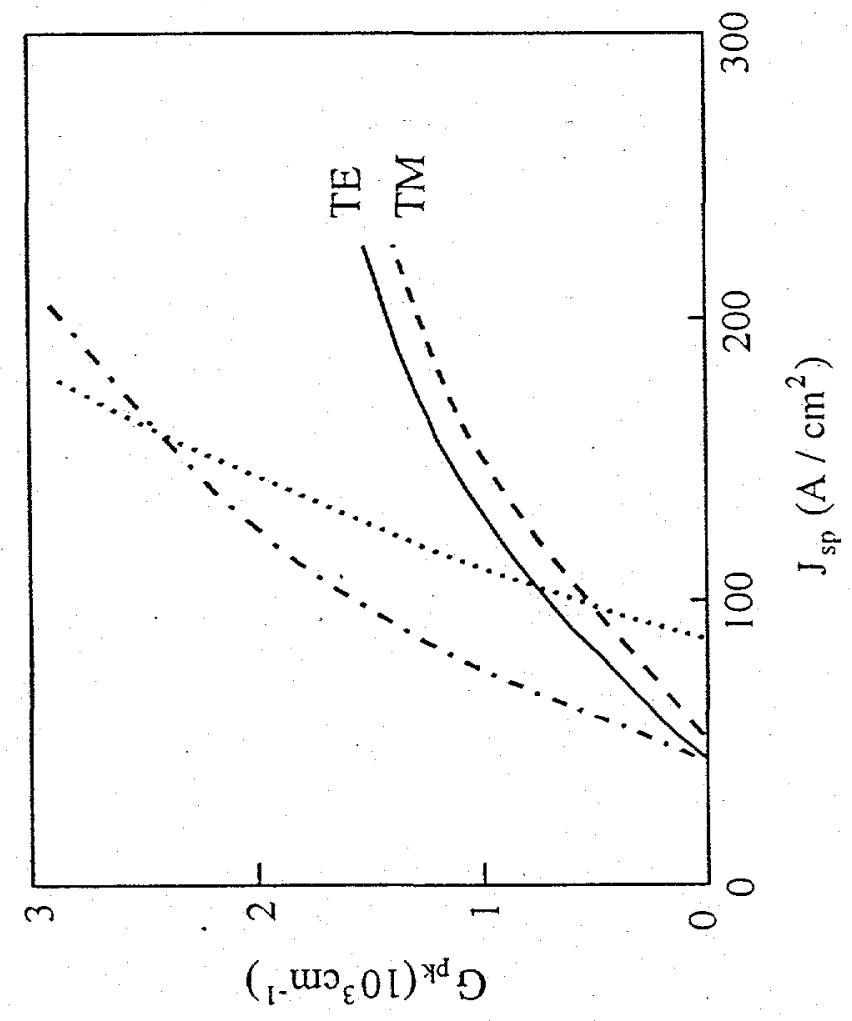

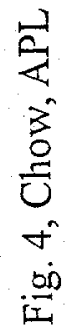

